\title{
Anomalous Josephson current via Majorana bound states in topological insulators
}

\author{
P. A. Ioselevich ${ }^{1,2}$ and M. V. Feigel'man ${ }^{1,2}$ \\ ${ }^{1}$ L. D. Landau Institute for Theoretical Physics, Kosygin str.2, Moscow 119334, Russia and \\ ${ }^{2}$ Moscow Institute of Physics and Technology, Moscow 141700, Russia
}

\begin{abstract}
We propose a setup involving Majorana bound states (MBS) hosted by a vortex on a superconducting surface of a 3D Topological Insulator (TI). We consider a narrow channel drilled across a TI slab with both sides covered by s-wave superconductor. In the presence of a vortex pinned to such a channel, it acts as a ballistic nanowire connecting the superconducting surfaces, with a pair of MBS localized in it. The energies of the MBS possess a $4 \pi$-periodic dependence on the superconductive phase difference $\varphi$ between the surfaces. It results in the appearence of an anomalous term in the current-phase relation, $I_{a}(\varphi)$ for the supercurrent flowing along the channel between the superconductive surfaces. We have calculated the shape of the $4 \pi$-periodic function $I_{a}(\varphi)$, as well as the dependence of its amplitude on temperature and system parameters.
\end{abstract}

Since Majorana bound states (MBS) were predicted to exist in solid state systems, a number of different suggestions on how to detect them has been made. MBS are predicted to exist in systems characterized by both strong spin-orbit coupling and superconductivity. Examples include the surface of a topological insulator (TI) covered by s-wave superconductor with vortices 1] or electrostatic defects [2], as well as ordinary semiconductor nanowires with spin-orbit coupling and proximityinduced superconductivity in the presence of a sufficiently strong Zeeman field [3]. While there are numerous suggestions on detection of MBS [1, 4 7], no experimental success has been reported yet. One more way to track down MBS in a superconducting proximity system is to observe an anomalous $4 \pi$-periodic phase dependence of a supercurrent. It was shown by A.Kitaev [8], that a specific 1-D fermionic chain hosting a pair of MBS switches its ground state fermionic parity under an adiabatic change of the superconducting phase difference $\varphi$ by $2 \pi$. Thus, if the chain conserves fermionic parity, its behavior has to be $4 \pi$-periodic.

In the present letter we propose and study a system with rather simple geometry, based on a TI sample covered by a superconducting film, and calculate the anomalous $4 \pi$-periodic supercurrent it carries. More specifically, we consider a flat thin slab of strong TI with both its surfaces covered by an s-wave superconducting film. The slab, together with the superconductive films, is pierced by a cylindric hole of radius $R$, see Fig.1. A single superconductive vortex with a flux quantum $\Phi_{0}=h c / 2 e$ is pinned to that hole. The two superconducting surfaces are connected far away from the hole, forming an SNS circuit with the hole acting as a normal region. Below we demonstrate that MBS should be present in this setup, and calculate the anomalous $4 \pi$-periodic component $I_{a}(\varphi)$ of the current flowing along the cylindric hole between the superconductive surfaces. Related issues were considered in a recent preprint [9] in terms of the Hopf invariant and its relation to fermionic parity. Note that in the SNS-like setup we consider, a Hopf invariant cannot be defined since a part of the TI surface is not

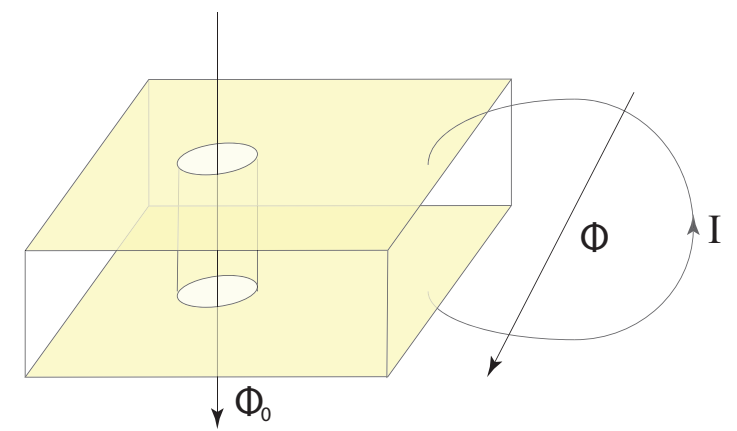

Figure 1: The system. A layer of TI has both surfaces covered by superconductor. A hole in the layer hosting a vortex forms an SNS-junction between the surfaces. The superconducting surfaces close away from the hole, completing an SNS-circuit with supercurrent flowing through the hole.

gapped.

In the simplest case of strong TI, realized in $\mathrm{Bi}_{2} \mathrm{Se}_{3}$ and $B i_{2} \mathrm{Te}_{3}$, surface electrons are described by a single Dirac cone with the Hamiltonian $H=v_{f} \sigma \cdot \mathbf{p}-E_{f}$, where $\sigma$ is the spin operator and $E_{f}$ is the Fermi energy of the surface states of the TI. Below we consider the semiclassical limit $\Delta \ll E_{f}$ and put $\hbar=1$. For the upper and lower surfaces covered by s-wave superconductor, a pairing term $\Delta \psi^{\dagger} \psi^{\dagger}+$ h.c. arises due to proximity effect [1]:

$\hat{H}=\left(v_{f} \sigma \cdot \mathbf{p}-E_{f}\right) \tau_{z}+\Delta(\mathbf{r})\left(\tau_{x} \cos \varphi(\mathbf{r})+\tau_{y} \sin \varphi(\mathbf{r})\right)(1)$

The Pauli matrices $\tau$ act in the Nambu-Gor'kov space, $\hat{K}$ denotes complex conjugation. For the Hamiltonians $H_{u, l}$ acting on upper and lower surfaces, one should replace $\Delta(\mathbf{r})$ and $\varphi(\mathbf{r})$ in Eq.(1) with $\Delta_{u, l}(\mathbf{r})$ and $\varphi_{u, l}(\mathbf{r})$ correspondingly. The operator $\hat{H}$ anticommutes with the electron-hole conjugation operator $\Xi=\sigma_{y} \tau_{y} \hat{K}$; we emphasize that this property holds for any Bogolyubov-De Gennes Hamiltonian with a most general form of singleelectron spectrum, including terms that break timeinversion symmetry. The symmetry $\{\hat{H}, \hat{\Xi}\}=0$ divides the eigenstates of the Hamiltonian (11) into a set of conjugate pairs $\psi_{E}, \psi_{-E}=\Xi \psi_{E}$ with opposite energies 
$(E,-E)$ and, possibly, a number of self-conjugate states with zero energy. In the basis of self-conjugated states all matrix elements of (1) are purely imaginary. The Hermitian matrix $H$ is antisymmetric in this basis, therefore the corresponding Pfaffian Pf $H$ can be defined. It is easy to see that generically the existence of Pf $H$ protects twofold degenerate zero-energy levels of $H$ against splitting under adiabatic variations of $H$. Indeed, consider a Hamiltonian with a parametric dependence $H(\varphi)$ such that it possesses a pair of zero eigenstates at some $\varphi=\varphi_{0}$. Then the determinant of the matrix $H$ has a double zero at $\varphi_{0}$, i.e. $(\operatorname{Pf} H)^{2}=\operatorname{det} H(\varphi) \propto\left(\varphi-\varphi_{0}\right)^{2}$, thus Pf $H(\varphi) \propto \varphi-\varphi_{0}$. Any small perturbation of the Hamiltonian (assuming it obeys the symmetry condition $\{\hat{H}, \hat{\Xi}\}=0$ ) can only shift the value of the crossing point $\varphi_{0}$, but its very existence is robust. Apparently, the above arguments are in contradiction with well-known properties of Andreev levels in a usual superconductive quantum point, with energies given [14] by $E_{ \pm}= \pm \Delta \sqrt{1-\mathcal{T} \sin ^{2} \frac{\varphi}{2}}$, so that an arbitrary small reflection probability $r=1-\mathcal{T}$ leads to the splitting of zero levels present at $\varphi=\pi$ in the ballistic case $\mathcal{T}=1$. The origin of this contradiction lies in the spin degeneracy present in usual systems without spin-orbit interaction: in the presence of such a degeneracy the Pfaffian of the ballistic contact has a double zero, $\operatorname{Pf} H(\varphi) \sim(\varphi-\pi)^{2}$, which is not robust to weak perturbations. Below we consider the generic case of strong spin-orbit coupling and thus no spin degeneracy.

The sign of Pf $H$ changes simulteneously with the fermionic parity $F_{0}$ of the global ground-state of the system [8, 9]. Indeed, the two eigenstates $|e\rangle,|o\rangle$ of the total Hamiltonian, which become degenerate when $E_{e, o}=0$ at $\varphi=\varphi_{0}$, have fermionic numbers differing by 1 . As the phase $\varphi$ passes $\varphi_{0}$, the roles of the ground state and the lowest excited state are interchanged. If the actual fermionic parity $F$ of the system cannot change due to conservation laws (which we will assume to be the case), we come to the following conclusion: each time a pair of Majorana levels crosses $E=0$, the ground-state $\left|g_{0}\right\rangle$ is transformed to the lowest excited state $\left|e_{0}\right\rangle$, and vice versa, $\left|e_{0}\right\rangle \rightarrow\left|g_{0}\right\rangle$. We argue now that while the phase $\varphi$ changes on the $(0,2 \pi)$ interval, an odd number of such crossings occurs, i.e. after a $2 \pi$ phase rotation our system does not return to its original state.

Consider first the system shown in Fig. 1 without the cylindric channel but with two point vortices present in both superconductive films, on the upper and lower surfaces. Each of them hosts a single MBS [1]. Due to finite thickness $L$ of the slab, these two MBS $\chi_{1,2}$ are hybridized into a single complex fermion $\psi=\chi_{1}+i \chi_{2}$. The energy $e_{0}(\varphi)$ of this fermionic mode is proportional, in general, to the amplitude of MBS tunnelling $\tilde{t} \sim e^{-L / \xi_{T I}}$ between the surfaces. However, for $\varphi=\pi$ the tunnelling amplitude vanishes due to destructive interference (see
Supplement 1), thus a single level crossing at $E=0$ occurs as $\varphi$ varies on the $(0,2 \pi)$ period. Let us now open the cylinder channel across the slab. It results in a drastic increase of hybridization between upper and lower superconductive surfaces, and in the appearence of an even (due to Kramers degeneracy) number of conductive modes. Since these additional modes appear in pairs only, the transformation $g_{0} \rightarrow e_{0}$ occurs on each $2 \pi$-period of $\varphi$ variation. The above arguments prove the existence of the anomalous component $I_{a}(\varphi)$ of the Josephson current which is odd under $2 \pi$-shift. Below we calculate its magnitude and temperature dependence.

To find the current flowing along the hole channel we first calculate the sub-gap spectrum of the contact. We assume that the tube's radius $R$ and length $L$ (the latter coincides with the thickness of our TI slab) satisfy the conditions

$$
p_{f}^{-1} \ll R, L \ll \xi_{0}, \xi_{s c}
$$

where $\xi_{0}=v_{f} / \Delta, p_{f}$ is the Fermi momentum of surface electrons of the TI, and $\xi_{s c}=\sqrt{D / 2 \Delta} \ll \xi_{0}$ is the actual "dirty-limit" coherence length in the superconductive film with diffusion coefficient $D$. Inequlities (2) mean, in particular, that we consider a short SNS-contact with many transverse channels, $N_{c h} \sim p_{f} R$. To find the sub-gap spectrum we solve Bogolyubov-de Gennes equations on both surfaces of the TI in the presence of the induced gap and vortices, and match obtained solutions with eigenmodes living on the inner cylindric surface of the channel.

We use cylindric coordinates $r, \theta, z$ with the $z$-axis coinciding with the tubes axis. The Hamiltonian (10) in the presence of an Abrikosov vortex is:

$$
\hat{H}=v_{f} \sigma \cdot\left(\mathbf{p} \tau_{z}-\nabla \theta / 2\right)-E_{F} \tau_{z}+\Delta(\mathbf{r}) \tau_{x}
$$

The magnetic screening length is very long for thin films, and the flux of the vortex is distributed over a radius much greater than both $R$ and $\xi_{0}$, thus we may neglect the vector-potential term in (3). For a fixed angular momentum $\nu$ we get $\Psi=e^{i \nu \theta-i \sigma_{z} \theta / 2}\left(\begin{array}{l}u(r) \\ v(r)\end{array}\right)$ with radial wave functions $u(r)$ and $v(r)$ given by

$$
\left(\begin{array}{cc}
\hat{H}_{\nu-N / 2}-\epsilon & \Delta(r) \\
\Delta(r) & -\hat{H}_{\nu+N / 2}-\epsilon
\end{array}\right)\left(\begin{array}{l}
u \\
v
\end{array}\right)=0
$$

with $\hat{H}_{m}$ standing for $v_{f}\left(\sigma_{x}\left(p_{r}-\frac{i}{2 r}\right)+\sigma_{y} \frac{m}{r}\right)-E_{f}$. The profile of the gap function near the vortex center is $\Delta(r) \approx r \Delta / \sqrt{r^{2}+2 \xi_{s c}^{2}}$. Equation (4) can be solved for 
$\epsilon \ll \Delta[10]$. In the first order in $\epsilon / \Delta$ it yields:

$$
\begin{array}{r}
u=\left[c_{1} e^{-i \phi} w_{\nu-1 / 2}^{(1)}(r)-i c_{2} e^{i \phi} w_{\nu-1 / 2}^{(2)}(r)\right] e^{-K} \\
v=\left[-i c_{1} e^{i \phi} w_{\nu+1 / 2}^{(1)}(r)+c_{2} e^{-i \phi} w_{\nu+1 / 2}^{(2)}(r)\right] e^{-K} \\
K(r)=\int_{0}^{r} \Delta(\rho) d \rho \\
\phi(r)=e^{2 K(r)} \int_{r}^{\infty}\left(\epsilon+\frac{\nu \Delta(\rho)}{p_{f} \rho}\right) e^{-2 K(\rho)} d \rho \\
w_{m}^{(1,2)}(r)=e^{ \pm \frac{i m}{2}}\left(\begin{array}{c}
H_{m-1 / 2}^{(1,2)}\left(p_{f} r\right) \\
i H_{m+1 / 2}^{(1,2)}\left(p_{f} r\right)
\end{array}\right)
\end{array}
$$

$H^{(1,2)}$ in (9) denote Hankel functions. When $\nu, \epsilon=0$, solutions (5), (6) become exact [1]. Due to the $e^{-K}$ factor in Eqs.(15), (6) , the wavefunction $\Psi$ is localized in the vicinity of the tube within a length $\sim \xi_{0}$. Next we specify electronic eigenmodes in the channel. The Hamiltonian for a cylindrical surface has the form 12 ]

$$
H_{c}=\left(\sigma_{z} p_{z}+\sigma_{\theta} p_{\theta}+\frac{i}{2 R} \sigma_{r}\right)
$$

with $\sigma_{\theta}=-\sigma_{x} \sin \theta+\sigma_{y} \cos \theta$ and $\sigma_{r}=\sigma_{x} \cos \theta+\sigma_{y} \sin \theta$. The eigenfunctions of (10) are

$$
\psi=e^{i p z} e^{i \theta \mu} e^{-i \sigma_{z} \theta / 2}\left(\begin{array}{c}
\cos \frac{\alpha}{2} \\
i \sin \frac{\alpha}{2}
\end{array}\right)
$$

where $\alpha=\arctan \frac{\mu}{R p}$. The energy spectrum for positive energies (counted from $E_{F}$ ) is $\epsilon_{\mu, \mathbf{p}}=\sqrt{p^{2}+\frac{\mu^{2}}{R^{2}}}$. The angular momentum is half-integer, $\mu=\frac{1}{2}+n$, due to the Berry phase originating from the rotation of the spin. We neglect the effect of the small magnetic flux $\Phi_{R} \ll$ $\Phi_{0}$ inside the cylinder. Note that the possibility to find conductive channels with no magnetic flux inside is due to the semiclassical condition $p_{f} R \gg 1$; on the contrary, in the ultraquantum limit $p_{f} R \leq 1$ the presence of a $\Phi_{0}$ flux would be necessary to make the hole conductive, see Supplement to Ref. [13].

Now we have to match wavefunctions (5), (6) on the upper and lower surfaces with wavefunctions (11) (and their analog for the hole component with $\epsilon<0$ ) on the boundaries between the cylinder and flat surfaces. The proper matching condition for a sharp edge reads:

$$
\Psi_{1}=\exp \left[-i \frac{\theta}{2}\left(\sigma \cdot \mathbf{n}_{\theta}\right)\right] \Psi_{2}
$$

Here $\mathbf{n}_{\theta}$ is the unit vector in the direction of the edge and $\theta$ is the rotation angle (equal to $\frac{\pi}{2}$ in our case). $\Psi_{1,2}$ are 4-component vector wavefunctions on both sides of the edge. The operator in Eq.(12) rotates the spin by the angle $\theta$ around the direction $\mathbf{n}_{\theta}$. We provide an explicit form of the matching equations and the derivation of the resulting energy spectrum in the Supplements 2,3.

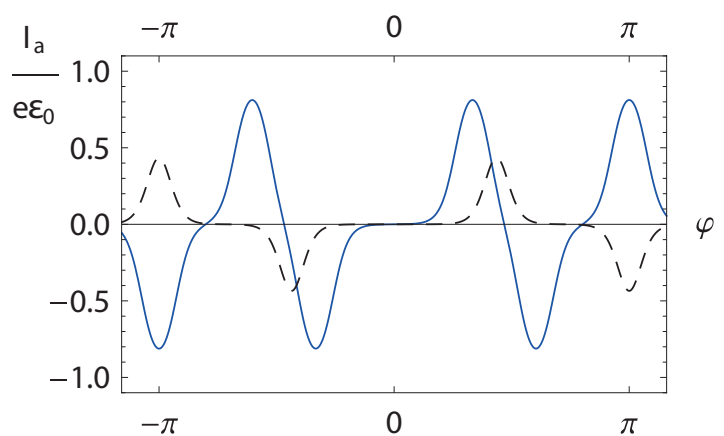

Figure 2: The anomalous current $I_{a}(\varphi)$ is computed for $p_{f} R=2$ (dashed line) and $p_{f} R=3$ (blue line). Other parameters are fixed as $p_{f} \xi=10, \quad p_{f} \xi_{s c}=5, \quad p_{f} L=6, \quad T=$ $0.05 \Delta$. The extrema of $I_{a}(\varphi)$ occur whenever a pair of conjugated levels crosses at $\epsilon=0$.

The resulting low-lying levels with $\epsilon \ll \Delta$ as function of the phase difference $\varphi$ are given by (up to the neglected terms of the order of $\left.\delta \varepsilon \sim \nu \Delta /\left(p_{f} R\right)^{2}\right)$ :

$$
\begin{array}{r}
\epsilon_{\nu k}(\varphi)= \pm \varepsilon_{0}(\varphi-\pi-2 \pi k)- \\
-\varepsilon_{1}\left[\arcsin \frac{\nu}{p_{f} R}+\frac{\nu L}{2 p_{f} R^{2}}\left(1-\frac{\nu^{2}}{\left(p_{f} R\right)^{2}}\right)^{-1 / 2}\right]
\end{array}
$$

where $\varepsilon_{1} \approx \Delta$ and $\varepsilon_{0} \approx \Delta / 2$ for the case $\xi_{s c} \ll \xi_{0}$ we consider, $|\nu|<p_{f} R$ and $k$ is an integer. No propagating modes in the tube exist at $\nu>p_{f} R$, instead there is an exponentially small overlap between the bound states residing inside vortex cores on opposite surfaces. The energies of the global eigenstates with $|\nu|>p_{f} R$ are given by $\epsilon_{\nu}=\nu \frac{\Delta}{p_{f} \xi_{0}} \log \frac{\xi_{0}}{\xi_{s c}}$ up to exponentially weak $\varphi$-dependent corrections. The result (13) is not applicable in the region $\left|\nu-p_{f} R\right| \sim\left(p_{f} R\right)^{1 / 3}$ where the crossover between hybridized and non-hybridized levels occurs.

The supercurrent through a short SNS-contact in thermal equilibrium can be expressed [14] in terms of Andreev levels: $I(\varphi)=-2 e \sum_{j: \epsilon_{j}>0} \tanh \left(\frac{\epsilon_{j}}{2 T}\right) \frac{\partial \epsilon_{j}}{\partial \varphi}$. Here all eigenstates are taken into account, regardless of their parity. The total current scales with the number of conductive channels in the hole: $I \sim e \Delta p_{f} R / \hbar$. To reveal any parity-related effects, we have to consider thermodynamic ensembles with odd and even numbers of quasiparticles separately. A standard route is to introduce thermodynamic potentials $\Omega_{\text {odd/even }}$, describing odd and even numbers of quasiparticles correspondingly [15]. Dividing the total current $I(\varphi)$ into a sum $I_{n}(\varphi)+I_{a}(\varphi)$ where $I_{n}(\varphi)$ is parity-independent, we obtain $I_{a}=(-1)^{F_{0}} e \frac{\partial}{\partial \varphi}\left(\Omega_{o d d}-\Omega_{\text {even }}\right)$, see Suppl.4. In terms of the spectrum of Andreev levels, it reads

$$
I_{a}(\varphi)=(-1)^{F_{0}} \frac{2 e f}{1-f^{2}} \sum_{j} \frac{1}{\sinh \frac{\epsilon_{j}}{T}} \frac{\partial \epsilon_{j}}{\partial \varphi}
$$

where $f=\prod_{j} \tanh \frac{\epsilon_{j}}{2 T}=f_{h y b} \cdot f_{\text {non }}$ and both the products and the sum are done over levels with $\epsilon_{j}>0$. Fac- 


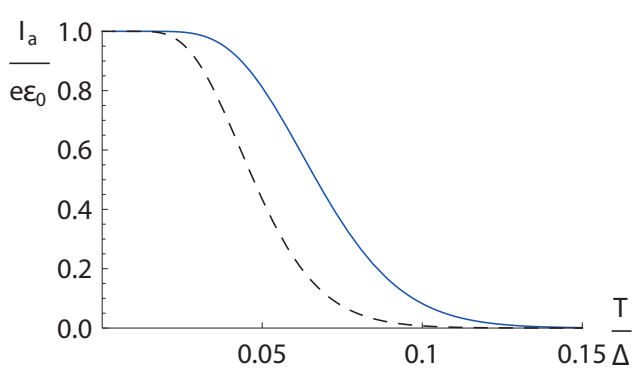

Figure 3: Temperature dependence of $I_{a}(\varphi=\pi)$ for the same sets of parameters as in Fig 2 Blue and dashed lines correspond to $p_{f} R=3$ and $p_{f} R=2$.

tors $f_{h y b}$ and $f_{\text {non }}$ correspond to the hybridized (currentcarrying) and non-hybridized levels. The physical meaning of $I_{a}$ is the current difference between an odd and an even state of the system. Eqs. (13[14) constitute our major quantitative result. Two examples of the $I_{a}(\varphi)$ dependence computed using Eqs. (13)14 are presented in Fig.2 $I_{a}(\varphi)$ experiences $\approx 2 p_{f} R$ oscillations in the $(-\pi,+\pi)$ interval and has opposite signs at $\varphi= \pm \pi$ where the amplitude of $I_{a}(\varphi)$ is maximal. Note that approximations used to derive Eq. (13) may lead to a deformation of the $I_{a}(\varphi)$ dependence, leading to an inhomogeneous shift of its oscillating pattern by the amount $\sim\left(p_{f} R\right)^{-1}$. It should not affect, however, the maximal value $I_{a}^{\max }$ of the anomalous current. The temperature dependence of $I_{a}^{\max }$ is presented in Fig. 3 .

To analyze the temperature dependence of $I_{a}(\varphi)$ we consider Eq.(14) in several limits. In the range of $T$ much higher than the typical level spacing $\varepsilon_{1} / p_{f} R$, the subproduct $f_{h y b} \sim e^{-\pi^{2} T p_{f} R / 4 \varepsilon_{1}}$ (for the derivation of this and the following formulae see Supplement 4). If, in addition, $T \gg E_{0} \equiv \Delta \frac{R}{\xi_{0}} \ln \frac{\xi_{0}}{\xi_{s c}}$, then the subproduct $f_{\text {non }}$ is also small: $f_{\text {non }}=e^{-\pi^{2} T / 2 \delta}$, where $\delta=\left(\Delta / p_{f} \xi_{0}\right) \ln \frac{\xi_{0}}{\xi_{\text {sc }}}$ is the typical level spacing within a single vortex core. Thus $I_{a}$ decays exponentially with temperature at $T>T_{1}=$ $\min \left(\frac{\Delta}{p_{f} R}, \frac{\Delta R}{\xi_{0}} \ln \frac{\xi_{0}}{\xi_{s c}}\right)$. At lower temperatures $T \ll T_{1}$ we find $f_{\text {hyb }}=\tanh \left(\epsilon_{1} / 2 T\right)$, where $\epsilon_{1}(\varphi)$ is the lowest hybridized level, therefore

$$
\begin{array}{r}
I_{a}(\varphi) \simeq \frac{e \varepsilon_{0} \cdot f_{\text {non }}}{\cosh ^{2} \frac{\epsilon_{1}}{2 T}-f_{\text {non }}^{2} \sinh ^{2} \frac{\epsilon_{1}}{2 T}} \\
f_{\text {non }}=\exp \left[-\frac{4 e^{-E_{0} / T}}{1-e^{-\delta / T}}\right]
\end{array}
$$

Simple analysis of Eqs. (15/16) leads to the second characteristic temperature $T_{2}=E_{0} / \ln \left(p_{f} \xi_{0}\right)$. Depending on parameters, $T_{2}$ may be both higher and lower than $T_{1}$. In addition, we mention the existence of non-hybridized subgap states localized in superconductive films near vortex cores, which may lead to some suppression of the crossover temperature $T_{2}$; however, we do not expect their effect to be drastic. Finally, the usual parity-effect temperature $T_{3}=\Delta / \ln (\nu V \Delta)$, see Ref. 15], puts an additional restriction for the temperature region where an anomalous current could be observed.

Summarizing the above analysis, we find that for the anomalous current $I_{a}$ to be detectable, the following condition must be met:

$$
T \leq \min \left(\frac{\Delta}{p_{f} R}, \frac{2 \Delta R \ln \left(\xi_{0} / \xi_{s c}\right)}{\xi_{0} \ln \left(p_{f} \xi_{0}\right)}, \frac{\Delta}{\ln (\nu V \Delta)}\right)
$$

The temperature dependence of the anomalous current is shown in Fig 3 for a specific choice of parameters such that $T_{2}<T_{1}$.

To conclude, we proposed a setup using strong topological insulator covered by superconductive films, which allows the detection of Majorana bound states through the measurement of an anomalous $4 \pi$-periodic component $I_{a}$ of the Josephson current. The temperature dependence of the $I_{a}$ amplitude is calculated, and the conditions for the proposed effect to be observed are found.

We are grateful to L. B. Ioffe, D. A. Ivanov, A. Yu. Kitaev, J. E. Moore and P. M. Ostrovsky for numerous discussions and advises. This research was supported by the RFBR grant \# 10-02-00554 and by the RAS program "Quantum physics of condensed matter".

[1] L. Fu and C. L. Kane, Phys.Rev.Lett. 100, 096407 (2008).

[2] M. Wimmer, A.R. Akhmerov et al Phys. Rev. Lett. 105, 046803 (2010)

[3] J. D. Sau, S. Das Sarma et al arXiv:1006.2829 (2010)

[4] A. R. Akhmerov, J. Nilsson, and C. W. J. Beenakker arXiv:0903.2196 (2009)

[5] K. T. Law, P. A. Lee, and T.K. Ng Phys. Rev. Lett. 103, 237001 (2009)

[6] R. M. Lutchyn, J. D. Sau, and S. Das Sarma, Phys. Rev. Lett. 105, 077001 (2010)

[7] J. D. Sau, S. Tewari, and S. Das Sarma, arXiv:1004.4702 (2010)

[8] A. Kitaev, arXiv: 0010440v2, (2000)

[9] Y. Ran, P. Hosur, and A. Vishwanath, arXiv:1003.1964 (2010)

[10] N. B. Kopnin and M. M. Salomaa, Phys. Rev. B 44, 9667 (1991).

[11] I. M. Khaymovich, N. B. Kopnin et al Phys. Rev. B 79, 224506 (2009)

[12] Y. Zhang, Y. Ran, and A. Vishwanath Phys. Rev. B 79, 245331 (2009)

[13] P. M. Ostrovsky, I. V. Gornyi, and A. D. Mirlin, Phys. Rev. Lett. 105, 036803 (2010).

[14] C. W. J. Beenakker and H. van Houten, Phys. Rev. Lett. 66, 23 (1991)

[15] M. T. Tuominen, M. Tinkham et al, Phys. Rev. Lett. 69, 1997 (1992). 


\section{ONLINE SUPPLMENTARY MATERIAL}

\section{Degeneracy of MBS at $\varphi=\pi$}

Beside of the $\Xi$-symmetry inherent to Bogolyubov-de Gennes equation, our model has a symmetry that is the composition $U$ of rotation $R$ and time-reversal $\Theta$. Indeed, by rotating our system around an axis lying in the $z=0$ plane (the plane lying between the surfaces of our slab), we arrive at the same system as by conjugating $\Delta$, possibly with a phase shift. Selecting a gauge, in which the superconducting phase is given by $\arg \Delta(\theta, z=L / 2)=$ $\theta=\arg \Delta(\theta, z=L / 2)-\varphi$, we find that $U$ commutes with the BdG Hamiltonian if the axis of rotation forms a $\varphi / 2$ angle with the $x$-axis. The part of $R$ that acts on spin variables equals $-i\left[\sigma_{x} \cos \varphi / 2+\sigma_{y} \sin \varphi / 2\right]$, while $\Theta=$ $i \sigma_{y} K$, where $K$ stands for complex conjugation. Hence, $U \sim \sin \varphi / 2+i \sigma_{z} \cos \varphi / 2$. The first term commutes with $\Xi \equiv \sigma_{y} \tau_{y} K$, while the second term does not, in general. We see that $U$ commutes with $\Xi$ only if $\varphi=\pi$. The commutation relations $[H, U]=0 ;[U, \Xi]=0 ;\{H, \Xi\}=0$ lead to the degeneracy of the $E=0$ levels of our system at $\varphi=\pi$.

\section{The matching equations}

Consider two surfaces -1 and 2 - joining on a line at the angle of $\theta_{0}$. Let $\Psi_{1,2}$ be the wave function on the edge of surface 1 and 2 respectively. We replace the sharp boundary by a smooth cylindric transition with a radius $r$. We use the natural cylindric coordinates of this cylindric sector with $\theta=0$ at its boundary with surface 1. Next we decompose $\Psi_{1,2}$ into the set of functions (11):

$$
\begin{array}{r}
\Psi_{1}=\sum_{p, \mu} a_{p, \mu} \Psi_{p, \mu} \\
\Psi_{2}=\sum_{p, \mu} a_{p, \mu} e^{i \theta \mu-i \sigma_{z} \theta_{0} / 2} \Psi_{p, \mu}
\end{array}
$$

with $\Psi_{p, \mu}=e^{i p z}\left(\begin{array}{c}\cos \frac{\alpha}{2} \\ i \sin \frac{\alpha}{2}\end{array}\right)$. In the limit $r \rightarrow 0$ we have to choose $\mu=0$ since $\sqrt{p^{2}+\frac{\mu^{2}}{r^{2}}}=\epsilon_{p, \mu}=$ const. This allows us to write $\Psi_{2}=e^{-i \sigma_{z} \theta_{0} / 2} \Psi_{1}$. The basis-independent form of this equation is given by Eq.(12).

\section{The spectrum equations}

The set of equations defining our systems spectrum consists of 8 equations - two sets of 4 matching equations corresponding to the two ends of the cylindric hole. To derive these equations, let we first write down the wave functions for some fixed momentum $\nu$; we will do it in linear approximation in small parameter $\frac{1}{p_{f} R} \ll 1$. The fixed angular momentum $\nu$ on the surfaces corresponds to $\nu_{ \pm}=\nu \pm 1 / 2$ in the channel: electron waves have $\mu=\nu_{-}$, while hole waves have $\mu=\nu_{+}$. After we have chosen the proper angular momenta, we only need to match wave functions at some fixed angle, say $\theta=0$. The w.f. in the cylinder is a superposition of four waves, representing electrons and holes propagating up and down the tube: $\Psi_{c y l}=\left(a_{\uparrow} u_{\uparrow}+a_{\downarrow} u_{\downarrow} b_{\uparrow} v_{\uparrow}+b_{\uparrow} v_{\uparrow}\right)^{T}$.

$$
\begin{array}{r}
u_{\uparrow}=e^{i\left(p_{f}+q\right) z \cos \alpha_{-}}\left(\begin{array}{c}
\cos \alpha_{-} / 2 \\
i \sin \alpha_{-} / 2
\end{array}\right) \\
u_{\downarrow}=e^{-i\left(p_{f}+q\right) z \cos \alpha_{-}}\left(\begin{array}{c}
-i \sin \alpha_{-} / 2 \\
\cos \alpha_{-} / 2
\end{array}\right) \\
v_{\downarrow}=e^{i\left(p_{f}-q\right) z \cos \alpha_{+}}\left(\begin{array}{c}
\cos \alpha_{+} / 2 \\
i \sin \alpha_{+} / 2
\end{array}\right) \\
v_{\uparrow}=e^{-i\left(p_{f}-q\right) z \cos \alpha_{+}}\left(\begin{array}{c}
-i \sin \alpha_{+} / 2 \\
\cos \alpha_{+} / 2
\end{array}\right) \\
\alpha_{ \pm}=\arcsin \frac{\nu \pm 1 / 2}{p_{f} R} \\
q \equiv \frac{\epsilon}{v_{f}}
\end{array}
$$

Next we write out the surface wave functions. At $r \ll \xi$ the solution (9) transforms into

$$
\begin{gathered}
u=c_{1} e^{-i \phi_{0}} w_{\nu-1 / 2}^{(1)}\left(r_{+}\right)-i c_{2} e^{i \phi_{0}} w_{\nu-1 / 2}^{(2)}\left(r_{+}\right) \\
v=-i c_{1} e^{i \phi_{0}} w_{\nu+1 / 2}^{(1)}\left(r_{-}\right)+c_{2} e^{-i \phi_{0}} w_{\nu+1 / 2}^{(2)}\left(r_{-}\right)
\end{gathered}
$$

with $r_{ \pm}=r\left(1 \pm q / p_{f}\right)$ and $\phi_{0}=\phi(0) \simeq q \xi_{0} / 2+$ $\frac{\nu}{p_{f} \xi_{0}} \log \frac{\xi_{0}}{\xi_{s c}}$ in the dirty limit $\xi_{s c} \ll \xi_{0}$. Using the asymptotics of Hankel functions with large arguments, we find the w.f. on the upper surface at $r=R, z=L, \theta=0$, within $\sim 1 / p_{f} R$ accuracy (we neglect terms $\sim\left(p_{f} R\right)^{-2}$ ):

$$
\begin{array}{r}
\Psi_{u p}(R)=C_{1}\left(\begin{array}{c}
\exp \left[i \beta-2 i \phi_{0}+\varphi\right] \\
i \exp \left[-2 i \phi_{0}+\varphi\right] \\
1 \\
i \exp [-i \beta]
\end{array}\right)+ \\
+C_{2}\left(\begin{array}{c}
\exp \left[-i \beta+2 i \phi_{0}+\varphi\right] \\
i \exp \left[2 i \phi_{0}+\varphi\right] \\
1 \\
i \exp [i \beta]
\end{array}\right)
\end{array}
$$

with some coefficients $C_{1}, C_{2}$ and $\cos \beta=\nu / p_{f} R$. This formula is correct unless $\left|\nu-p_{f} R\right| \lesssim\left(p_{f} R\right)^{1 / 3}$. $\Psi_{\text {down }}(R)$ is obtained by introducing two new coefficients $Q_{1}, Q_{2}$ instead of $C_{1}, C_{2}$ and putting $\varphi=0$.

Now we can construct the matching equations according to the matching rule (12). Acting on $\Psi_{c y l}(z=L)$ and $\Psi_{c y l}(z=0)$ by $\frac{1}{\sqrt{2}}\left(1-i \sigma_{y}\right)$ and $\frac{1}{\sqrt{2}}\left(1+i \sigma_{y}\right)$ respectively we finally get 


$$
\begin{aligned}
A_{\uparrow} e^{i\left(p_{f}+q\right) L \cos \alpha_{-}}\left(\begin{array}{c}
1 \\
e^{i \alpha_{-}}
\end{array}\right)+A_{\downarrow} e^{-i\left(p_{f}+q\right) L \cos \alpha_{-}}\left(\begin{array}{c}
-e^{i \alpha_{-}} \\
1
\end{array}\right) & =C_{1}\left(\begin{array}{c}
\exp \left[i \beta-2 i \phi_{0}+\varphi\right] \\
i \exp \left[-2 i \phi_{0}+\varphi\right]
\end{array}\right)+C_{2}\left(\begin{array}{c}
\exp \left[-i \beta+2 i \phi_{0}+\varphi\right] \\
i \exp \left[2 i \phi_{0}+\varphi\right]
\end{array}\right) \\
B_{\downarrow} e^{i\left(p_{f}-q\right) L \cos \alpha_{+}}\left(\begin{array}{c}
1 \\
e^{i \alpha_{+}}
\end{array}\right)+B_{\uparrow} e^{-i\left(p_{f}-q\right) L \cos \alpha_{+}}\left(\begin{array}{c}
-e^{i \alpha_{+}} \\
1
\end{array}\right) & =C_{1}\left(\begin{array}{c}
1 \\
i \exp [-i \beta]
\end{array}\right)+C_{2}\left(\begin{array}{c}
1 \\
i \exp [i \beta]
\end{array}\right) \\
A_{\uparrow}\left(\begin{array}{c}
e^{i \alpha_{-}} \\
-1
\end{array}\right)+A_{\downarrow}\left(\begin{array}{c}
1 \\
e^{i \alpha_{-}}
\end{array}\right) & =Q_{1}\left(\begin{array}{c}
\exp \left[i \beta-2 i \phi_{0}\right] \\
i \exp \left[-2 i \phi_{0}\right]
\end{array}\right)+Q_{2}\left(\begin{array}{c}
\exp \left[-i \beta+2 i \phi_{0}\right] \\
i \exp \left[2 i \phi_{0}\right]
\end{array}\right) \\
B_{\downarrow}\left(\begin{array}{c}
e^{i \alpha_{+}} \\
-1
\end{array}\right)+B_{\uparrow}\left(\begin{array}{c}
1 \\
e^{i \alpha_{+}}
\end{array}\right) & =Q_{1}\left(\begin{array}{c}
1 \\
i \exp [-i \beta]
\end{array}\right)+Q_{2}\left(\begin{array}{c}
1 \\
i \exp [i \beta]
\end{array}\right)
\end{aligned}
$$

Since $\alpha_{ \pm}=\pi / 2-\beta$ within our approximation, the spinors with coefficients $A_{\uparrow}$ and $C_{1}$ in the first equation of the system (28) are collinear. The same is true for the spinors with coefficients $A_{\downarrow}$ and $C_{2}$ in the same equation. A similar statement is true for each of the other Eqs.(28). Consequently, the system (28) splits into two simple subsystems:

$$
\begin{aligned}
A_{\uparrow} & =C_{1} \exp \left[i \beta-2 i \phi_{0}+i \varphi-i\left(p_{f}+q\right) L \cos \alpha_{-}\right] \\
B_{\downarrow} & =C_{1} \exp \left[-i\left(p_{f}-q\right) L \cos \alpha_{+}\right] \\
A_{\uparrow} & =Q_{2} \exp \left[-i \pi / 2+2 i \phi_{0}\right] \\
B_{\downarrow} & =Q_{2} \exp [-i \pi / 2+i \beta]
\end{aligned}
$$

and

$$
\begin{aligned}
A_{\downarrow} & =C_{2} \exp \left[i \pi / 2+2 i \phi_{0}+i \varphi+i\left(p_{f}+q\right) L \cos \alpha_{-}\right] \\
B_{\uparrow} & =C_{2} \exp \left[i \pi / 2+i \beta+i\left(p_{f}-q\right) L \cos \alpha_{+}\right] \\
A_{\downarrow} & =Q_{1} \exp \left[-2 i \phi_{0}+i \beta\right] \\
B_{\uparrow} & =Q_{1}
\end{aligned}
$$

These equations give the following set of the two spectral equations

$$
\begin{array}{r}
4 \phi_{0}=-2 \alpha \pm(\varphi-\pi-2 \pi k)+ \\
+\left(p_{f}-q\right) L \cos \alpha_{+}-\left(p_{f}+q\right) L \cos \alpha_{-}
\end{array}
$$

where the upper/lower signs refer to the systems (29) and (30) correspondingly. Since we assume $L \ll \xi_{0}$ and $R \ll \xi$, we neglect the $\sim q L$ term in the r.h.s of (31) and the $\nu$-dependent term in $\phi_{0}$, arriving at the final formula (13).

\section{The anomalous current}

The thermodynamic potential of a system with a fixed parity can be written as

$$
\Omega_{\text {odd } / \text { even }}=-T \ln \frac{\prod_{i}\left(1+e^{-\beta \epsilon_{i}}\right) \mp \prod_{i}\left(1-e^{-\beta \epsilon_{i}}\right)}{2}
$$

with $\beta=T^{-1}$. The difference $\delta \Omega=\Omega_{\text {odd }}-\Omega_{\text {even }}$ equals

$$
\delta \Omega=-T \ln \frac{1-f}{1+f} \quad \text { with } \quad f \equiv \prod_{i} \tanh \frac{\beta \epsilon_{i}}{2}
$$

Hence

$$
I_{a}=e \delta \Omega_{\varphi}^{\prime}=\frac{2 T e f_{\varphi}^{\prime}}{1-f^{2}}=\frac{2 T f e}{1-f^{2}} \sum_{i} \frac{\beta \epsilon_{i, \varphi}^{\prime}}{2 \cosh ^{2} \frac{\beta \epsilon_{i}}{2} \tanh \frac{\beta \epsilon_{i}}{2}}=\frac{2 f e}{1-f^{2}} \sum_{i} \frac{\epsilon_{i, \varphi}^{\prime}}{\sinh \beta \epsilon_{i}}
$$

It is useful to divide $f$ into factors corresponding to the hybridized and non-hybridized parts of the discrete spectrum.

$$
f=\prod_{|\nu|<p_{f} R} \tanh \frac{\epsilon_{\nu}}{2 T} \prod_{|\nu|>p_{f} R} \tanh \frac{\epsilon_{\nu}}{2 T}=f_{\text {hyb }} f_{\text {non }}
$$

We neglect the exponentially small dependence of nonhybridized energies on $\varphi$, so that

$$
I_{a}=\frac{2 e f_{\text {hyb }} f_{\text {non }}}{1-f_{\text {hyb }}^{2} f_{\text {non }}^{2}} J \quad J=\sum_{\left|\nu<p_{f} R\right|} \frac{\partial \epsilon_{\nu} / \partial \varphi}{\sinh \left(\epsilon_{\nu} / T\right)}(36)
$$

First let us analyze $f_{h y b}$. The typical level spacing is $\omega_{0} \sim \frac{\Delta}{p_{f} R}$. If $T \gg \omega_{0}$, we can write $f_{h y b}=$ $\exp \left[\int_{0}^{\infty} \ln \tanh \left(\frac{\omega_{0} \nu}{2 T}\right) d \nu\right]=\exp \left[-\frac{\pi^{2} T}{4 \omega_{0}}\right]$. For small $L / R$ 
and $\varphi=\pi$ this gives $\exp \left[-\frac{\pi^{2} p_{f} R T}{2 \Delta}\right]$ In the opposite case $T \ll \omega_{0}$ we can write $f_{h y b}=\tanh \frac{E_{h}}{2 T}$, where $E_{h}$ is the lowest hybridized energy level.

Next we consider factor $J$. At $T \gg \omega_{0}$ we use $\sinh (\epsilon / T) \simeq \epsilon / T$ at small energies and get $J \lesssim$ $\sum_{n=1}^{T / \omega_{0}} \frac{T \Delta}{n \omega_{0}}=\frac{T \Delta}{\omega_{0}} \log \frac{T}{\omega_{0}}$. At $T \ll \omega_{0}$ the amplitude of $J$ is dominated by the lowest hybridized level $\epsilon_{1}$ and we get $|J|=\frac{\Delta}{2 \sinh \epsilon_{1} / T}$.

Finally, we analyze $f_{n o n}$. The spectrum of the nonhybridized de Gennes states is described by $E_{\text {non }}=$ $\frac{\Delta}{p_{f} \xi} \ln \left(\frac{\xi_{0}}{\xi_{s c}}\right)\left[p_{f} R+n\right]=E_{0}+n \delta$ with $n=0,1,2 \ldots$ There are two copies of these series - one for the upper and one for the lower surface. If $\left(T-E_{0}\right) \gg \delta$ we can rewrite

$$
f_{\text {non }}=\exp \left[2 \int_{p_{f} R}^{\infty} \ln \tanh \frac{n \delta}{2 T} d n\right]=\exp \left[-\frac{\pi^{2} T}{2 \delta}-\frac{4 T}{\delta} \int_{0}^{\frac{E_{0}}{2 T}} \ln \tanh x d x\right]
$$

The second term can be neglected, if $T \gg E_{0}$. At low temperatures $T \ll E_{0}$ we have

$$
f_{\text {non }}=\exp \left[2 \sum_{n} \ln \left[1-2 e^{-\frac{E_{n}}{T}}\right]\right]=\exp \left[-4 e^{-\frac{E_{0}}{T}} \sum_{n=0}^{\infty} e^{-\frac{n \delta}{T}}\right]=\exp \left[-4 \frac{e^{-\frac{E_{0}}{T}}}{1-e^{-\frac{\delta}{T}}}\right]
$$

The low-temperature expressions derived for $f_{h y b}, f_{n o n}, J$ lead to formulae $(15,16)$. 